\title{
DISEÑO E IMPLEMENTACIÓN DE UN SISTEMA DE INFORMACIÓN PARA EL
} SEGUIMIENTO DE PROYECTOS DE SENTENCIAS EN EL TRIBUNAL DE LO CONTENCIOSO ADMINISTRATIVO

\section{DESIGN AND IMPLEMENTATION OF AN INFORMATION SYSTEM FOR THE FOLLOW-UP OF PROJECTS OF SENTENCES IN THE COURT OF ADMINISTRATIVE CONTENT}

Juan Manuel Fernández Álvarez ${ }^{1}$ Antonio Moreno Jauregui ${ }^{1}$

M. en C. Juan García Virgen ${ }^{2}$ M. en C. J. Reyes Benavides Delgado ${ }^{2}$

1. Estudiante de la Maestría en Sistemas Computacionales en la División de Estudios de Posgrado e Investigación Instituto Tecnológico de Colima (México.

E-mail: juangogof1@gmail.com ingantonmor@gmail.com

2. Profesor de la Maestría en Sistemas Computacionales en la División de Estudios de Posgrado e Investigación Instituto Tecnológico de Colima (México).

E-mail: jgarcia@itcolima.edu.mx, rbenavides@itcolima.edu.mx

E-mail de contacto: juangogof1@gmail.com

\section{Citación sugerida:}

Fernández Álvarez, JM., Moreno Juaregui, A., García Virgen, J. y Benavides Delgado, R. (2017). Diseño e implementación de un sistema de información para el seguimiento de proyectos de sentencias en el tribunal de lo contencioso administrativo. 3C TIC: Cuadernos de desarrollo aplicados a las TIC, 6(2), 13-27. DOI: <http://dx.doi.org/10.17993/3ctic.2017.56.13-27/>. 


\section{RESUMEN}

En esta investigación se desarrolló un sistema de información que da seguimiento a los proyectos de sentencias en un ambiente web gestionados por el Tribunal de lo Contencioso Administrativo para mejorar la impartición de justicia. El sistema prevé una mejora en la productividad de los procesos involucrados para el dictamen de sentencias, utilizando bitácoras para el registro histórico de las actividades incluyendo los tiempos respectivos de cada actividad involucrada durante el proceso y estableciendo puntos de verificación para conocer el avance del litigio.

\section{ABSTRACT}

In this research, was developed an information system to follow up the draft sentences in a web environment managed by the Administrative Contentious Court to improve the delivery of justice. The system provides for an improvement in productivity of the processes involved for the opinion of judgments, using logs for the historical record of the activities including their respective time for each activity used during the process and checkpoints were established to know the progress of the litigation.

\section{PALABRAS CLAVE}

Seguimiento de Proyecto de Sentencias, Aplicación Web, Dictamen de Sentencias, productividad en los procesos jurídicos.

\section{KEYWORDS}

Follow-up of Sentences Project, Web Application, Sentence Opinion, productivity in legal processes. 


\section{INTRODUCCIÓN}

Desde que se decretó, por medio del "Compromiso de Túnez" (Mundo, 2005), el uso de las TIC's en los procesos judiciales, se ha instituido un cambio dentro del Marco Jurídico Internacional y se ha buscado poder tener una implementación para proporcionar una mejor eficiencia, aunque esto ha sido un poco difícil, ya que al implementar estas tecnologías se minoriza el uso de la presencia de sujetos para algunas de las actuaciones del proceso. El Tribunal de lo Contencioso Administrativo del Estado de Colima (TCAEC, 1996) lleva una serie de procesos para poder llevar a juicio una demanda, determinar una sentencia y darle seguimiento: Cuando la demanda llega a manos del proyectista, este determina y justifica cual va a ser la sentencia que se va a realizar, que actualmente es un proceso ineficiente, ya que tiene que revisar los documentos del demandante, demandado y las leyes que intervienen en la promoción para poder determinar dicha sentencia. Después de realizar este análisis, la sentencia propuesta pasa a manos del magistrado, el cual también realiza la misma acción de leer los documentos y la justificación de la sentencia propuesta por el proyectista y determinar si esta procede o no y, después de haberse aceptado este veredicto, se le da seguimiento para comprobar que se haya cumplido por la parte demandada. En caso de no haberse cumplido el demandante podrá realizar una promoción para externar el no cumplimiento del mismo, así como también la parte demandada podrá hacer una promoción para un amparo. Ya que este proceso se lleva de una manera manual, lo cual resulta poco eficiente al momento de realizar la búsqueda y consulta de documentos, con el desarrollo del sistema se busca reducir ese tiempo y tener una recuperación inmediata de la información registrada.

Esta investigación es parte de un trabajo que integra tres etapas: almacenamiento y minería de la información, seguimiento de demandas y seguimiento de sentencias dentro del TCAEC, esta última etapa corresponde al trabajo de investigación que se describe en este documento, tal como se ilustra en la sección resaltada en rojo de la figura 1.

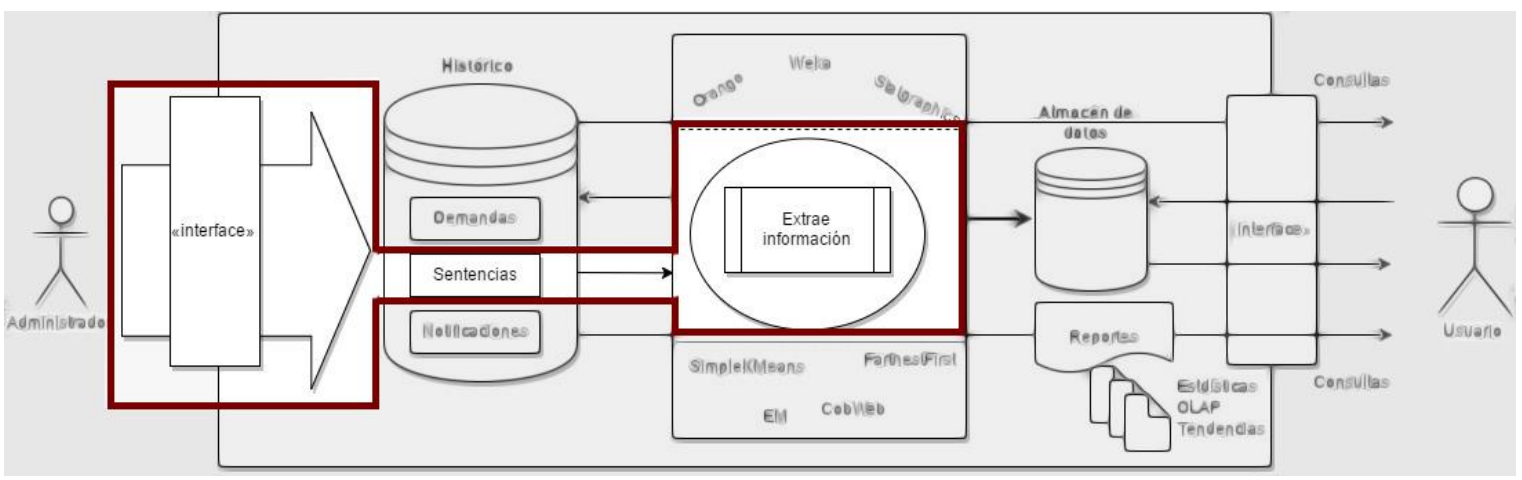

Figura 2. Modelo conceptual.

Urdapilleta, (2015), en su Artículo "Notas generales del Juicio en Línea” menciona también que el proceso jurídico que se sigue es el mismo de revisar cada una de las promociones, pruebas y actuaciones. También se siguen dictando las sentencias y acuerdos, la única diferencia es que ya no son documentos físicos con firmas autógrafas, sino que ya son documentos electrónicos con firma electrónica avanzada, por lo cual facilita la búsqueda de información y documentos y 


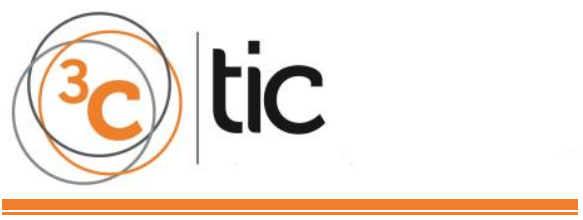

de seguimiento de expedientes, y propone que las dependencias correspondientes tomen la iniciativa para utilizar estas herramientas e implementarlas en los procesos que se siguen de cada uno de ellos. Gustavo Quintero Navas, en su artículo "Contencioso Administrativo y medios electrónicos: un gran paso hacia la modernización del ejercicio de la justicia administrativa" (Navas, 2011), brinda un enfoque general acerca de la importancia de la impleentación de las TIC's en el seguimiento de un proceso judicial electrónico y define algunas de las opciones y métodos que se pueden implementar para el buen funcionamiento de un Sistema de Seguimiento de este proceso. Brinda algunas herramientas y propone llevar un proceso de digitalización tanto de documentos como de firmas digitales, así como también notificaciones electrónicas. Una propuesta para mejora de esta investigación es indagar a fondo acerca del proceso de elaboración de cada uno de los acuerdos para llevar a cabo un buen seguimiento a este proceso, ya que el solo implemetar la digitalización es unicamente para tener en electrónico el expediente pero no muestra un seguimiento como tal del proceso juridico-administrativo.

Considerando las áreas de oportunidad detectadas en diferentes investigaciones revisadas en el punto anterior y habiendo analizado los proyectos ya implementados en algunas dependencias de otros estados, la más común, es que a pesar de tener un seguimiento de las demandas y sentencias dictadas, no se les da un seguimiento con alguna bitácora para determinar el tiempo en horas hombre en que dura el proceso de la redacción, validación y cumplimiento o no cumplimiento de cada una de ellas, por lo cual se propone desarrollar un sistema informático automatizado en un ambiente web, para mejorar el proceso del litigio.

\section{PROCESO DE DESARROLLO DEL SISTEMA}

Para el desarrollo de este proyecto se utilizó la metodología de Proceso Unificado Ágil (Métodos de desarrollo Ágil, 2016). A cada uno de los usuarios se elaboraron interfaces interactivas para que se desarrolle el proyecto de sentencia, se haga una revisión del mismo, y, una vez aprobado, se lleve a las firmas correspondientes de autorización para notificación a las partes involucradas. Cada uno de los diagramas se elaboró con la herramienta de desarrollo UML Enterprise Architect. La implementación de la base de datos se realizó con MySQL y para el desarrollo de las vistas se utilizó Codelgniter (Alejandro Morales Gámez, 2015), que es un framework PHP para el desarrollo rápido de aplicaciones web y ha permitido desarrollar el sistema web para el seguimiento de proyectos de sentencia en el Tribunal de lo Contencioso Administrativo del Estado de Colima. Para la elaboración de este proyecto se realizó una planeación para cada una de las fases de la metodología empleada, la cual se ilustra en la figura 2. En la Figura 3 se ilustra la arquitectura de Codeigniter, el modelo se refiere a todo el código que tiene que ver con el acceso a base de datos, en la vista codifica y mantiene la presentación final de nuestra aplicación de cara al usuario y en el controlador guardamos la lógica de nuestras páginas y realizamos todas las acciones que sean necesarias para generarlas, ayudados del modelo o la vista. 


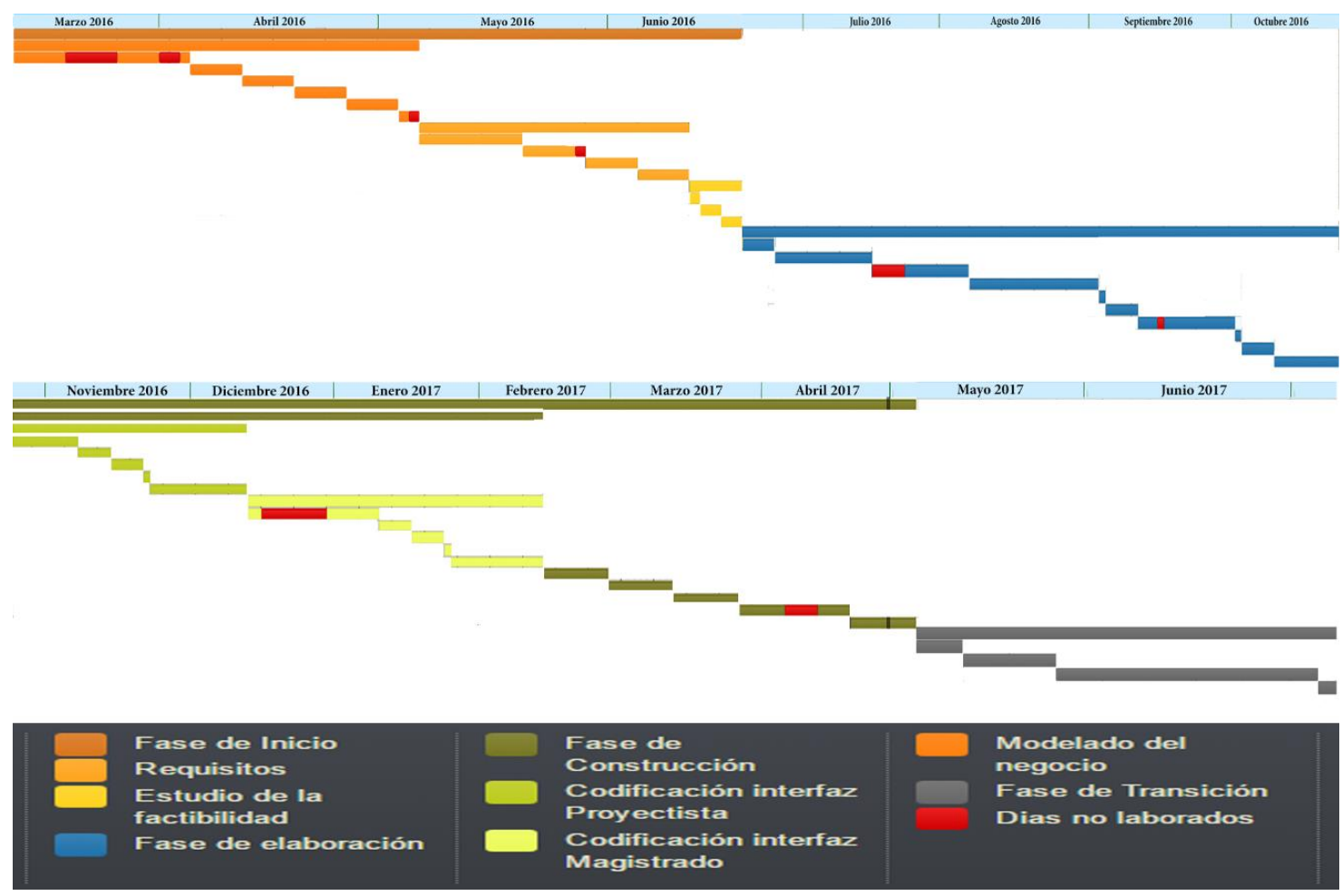

Figura 3. Gráfica de Gantt, Fases de desarrollo de SISESE.

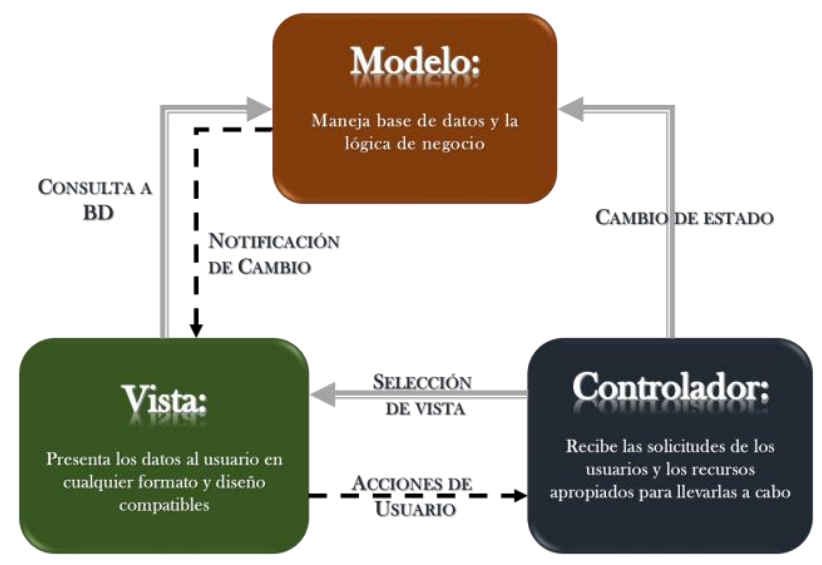

Figura 4. Arquitectura Modelo, Vista, Controlador de Codelgniter Fuente: Alejandro Morales Gámez, 2015.

Algunas de estas interfaces son utilizadas por los usuarios para la redacción, tanto del proyecto de sentencia, como su revisión y elaboración de diferentes tipos de acuerdos y/o documentos correspondientes a la sentencia, a continuación se muestra el desarrollo de la aplicación web. 


\section{DESARROLLO}

\subsection{ANÁLISIS}

En esta fase se detectaron cada una de los requerimientos para poder llevar a cabo la elaboración del sistema. Para ello, se hizo una entrevista con cada uno de los actores involucrados en el sistema, los cuales se ilustran en la figura 4.
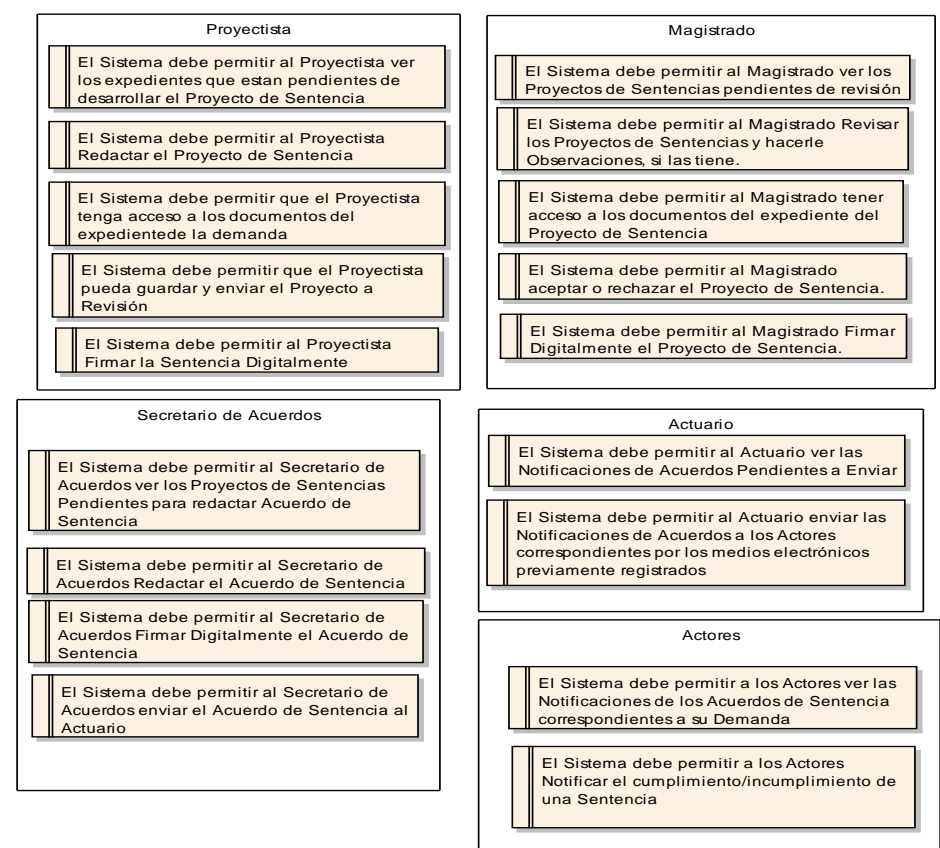

Figura 5. Requerimientos Funcionales.

\subsection{CASOS DE USO}

En la Figura 5 se ilustran los casos de uso general del Sistema SISESE. Cada uno de ellos nos muestra la interacción de cada uno de los usuarios con el Sistema, teniendo como usuarios principales al Proyectista, que es quien desarrolla el Proyecto de Sentencia y lo envía a ser evaluado para su aprobación o corrección. El Magistrado es el encargado de hacer esta evaluación del Proyecto de Sentencia.

Una vez aprobado entra en función el Secretario de acuerdos, en conjunto con el Proyectista y Magistrado proceden a firmar la Sentencia para poder pasar al Actuario, quien notifica a las partes involucradas en la Demanda, que es la Sentencia correspondiente a la misma. 


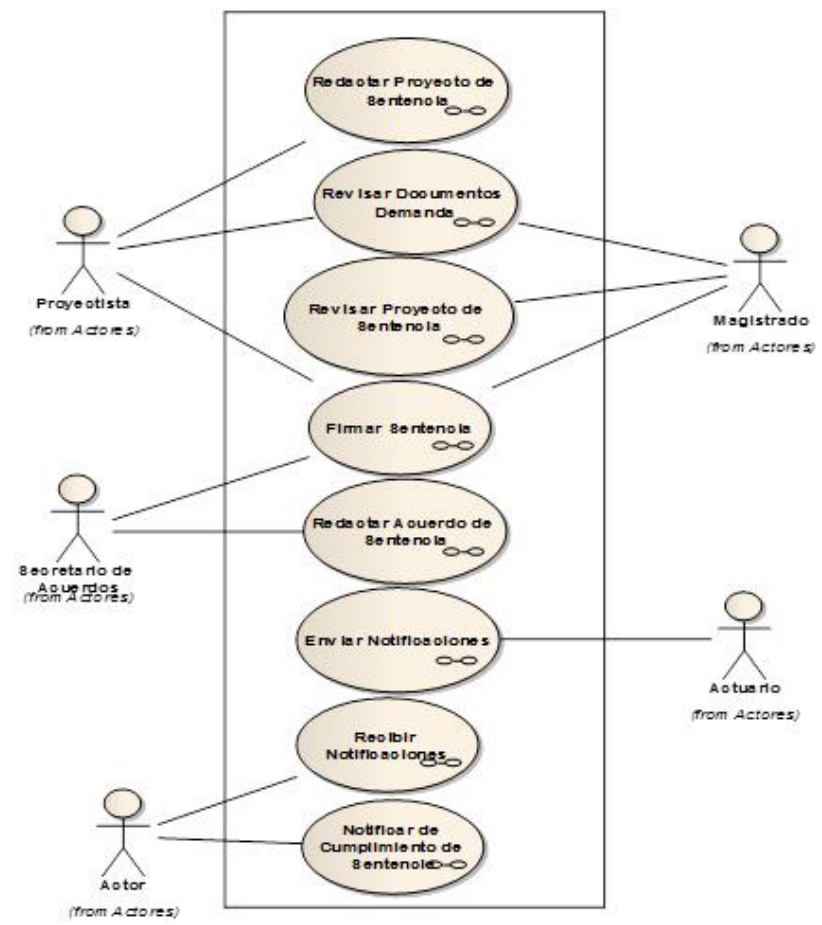

Figura 6.Diagrama de casos de uso SISESE.

\subsection{DISEÑO DE INTERFACES}

Para la navegación entre las interfaces de los usuarios se realizaron las interfaces con la herramienta Enterprise Architect 8.0, que fueron diseñadas para después ser codificadas. En la figura 5 se ilustra el mapa de navegación del Usuario Magistrado, el cual, después de tener acceso a su cuenta, puede revisar las propuestas de sentencias realizadas por cada uno de los Proyectistas y tiene la opción de leer los documentos que pertenecen a esa Demanda para validar la información. También puede hacerle modificaciones, si las requiere, aceptar el proyecto para enviarlo a los Actores involucrados o rechazar la propuesta para que se vuelva a realizar.

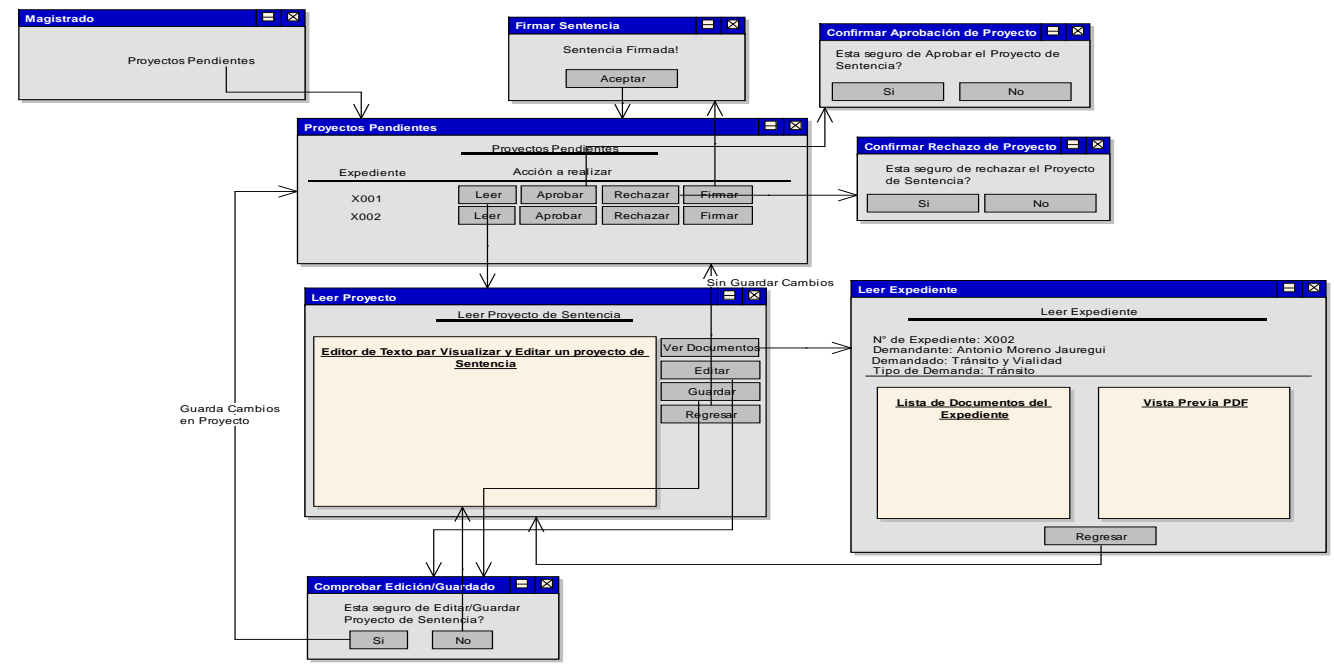

Figura 7. Diagrama de Navegación Magistrado. 


\subsection{CODIFICACIÓN DE INTERFACES (VISTAS)}

Para el desarrollo de cada una de las interfaces se utilizó el framework Codelgniter (Gámez, 2015), así como también el editor de texto Sublime para la realización de esta codificación.

En la figura 6 se ilustra el diseño de la Vista para redactar/editar los documentos que se desarrollan por parte del Proyectista, Magistrado y Actuario, y para poder realizar la redacción se implementó el editor de texto para php CKEditor en su versión completa.

En la figura 7 se ilustra el controlador utilizado para guardar el documento que se ha redactado/editado. El documento se guardará en una carpeta llamada Proyectos que tendrá el nombre del documento incluyendo el folio de la demanda.

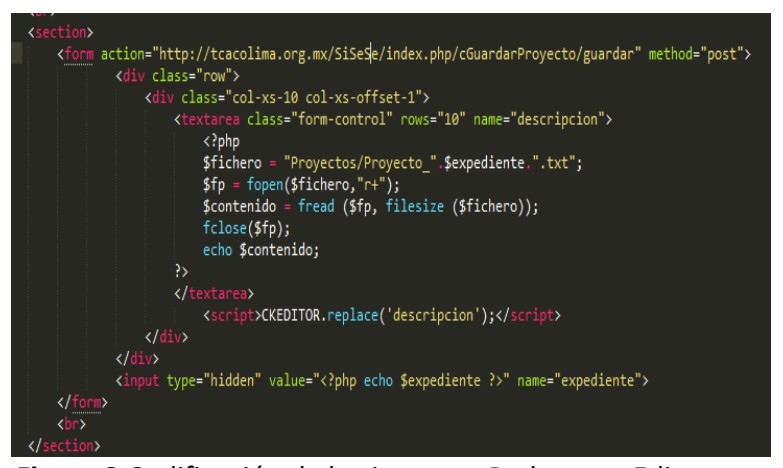

Figura 8.Codificación de la vista para Redactar o Editar un documento.

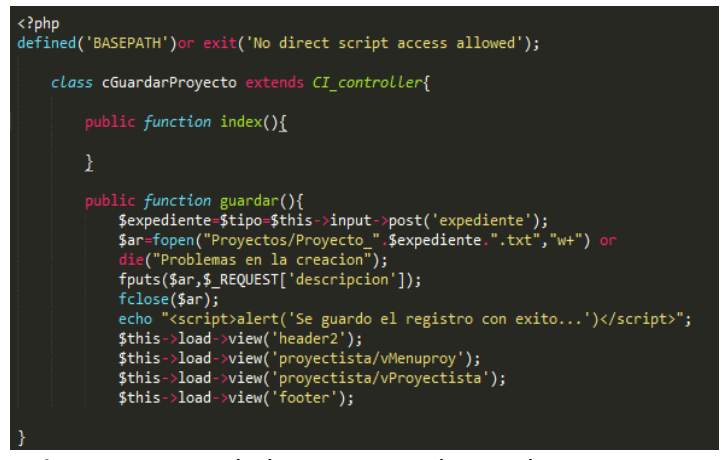

Figura 9. Controlador para guardar un documento.

\section{IMPLANTACIÓN}

La estrategia de implantación del sistema se determinó basándose en la información acumulada de las fases anteriores, en función del número de módulos del sistema de información implicados en la implantación, cuyo alcance depende de las características y complejidad de los sistemas de información.

El plan contempla las siguientes tareas, las cuales fueron nombradas como ISI (Implantación del Sistema de Información):

\section{Tarea ISI 1: En primer instancia de definió el plan de implantación}

Una vez analizada la información anterior, se desarrolló un plan de implantación que permita calcular adecuadamente el esfuerzo y los recursos necesarios para llevar a cabo con éxito la implantación.

Participantes: Equipo de usuarios, Administrador del sistema, Analista de Sistemas.

Responsable: Analista de sistemas. 


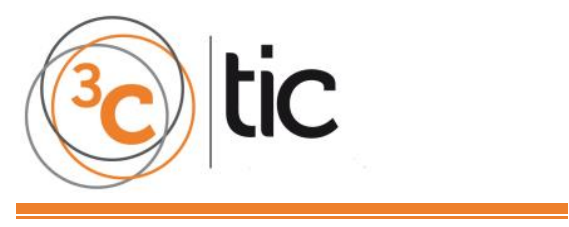

Para esta tarea se tomaron en cuenta los siguientes puntos:

- La capacitación necesaria para la implantación al equipo de trabajo que se encarga de realizar la implantación.

- La preparación de la infraestructura necesaria para la incorporación del sistema al entorno del proceso.

- La instalación de todos los componentes y procedimientos manuales y automáticos asociados a cada módulo del sistema de información implicados en la implantación.

- La ejecución de los procedimientos de carga inicial y migración de datos.

\section{Tarea ISI 2: Preparación del entorno del proceso}

El objetivo de la preparación del entorno del proceso es planificar que todos los recursos estén disponibles para la puesta en funcionamiento del Sistema de Información. Para esto se aseguró que hubiera disponibilidad del entorno y de los datos que se requieren para poner en ejecución el sistema.

Participantes: Administrador del sistema, Analista de Sistemas.

Responsable: Analista de sistemas.

\section{Tarea ISI 3: Capacitación para la implantación}

En esta tarea se dispone y se comparte la capacitación al equipo que participará en la implantación del sistema, y al personal de manejo del Sistema. Se realiza también el seguimiento de la capacitación de los usuarios finales para asegurar que la implantación se llevará a cabo correctamente.

Participantes: Usuarios del sistema, Administrador del sistema, Analista de Sistemas.

Responsable: Analista de sistemas.

\section{Tarea ISI 4: Presentación de procedimientos normativos}

Una vez que se ha realizado la evaluación de los procedimientos normativos en la fase de Construcción y Pruebas del Sistema, el Analista Funcional conjuntamente con el Líder y el Ejecutivo del Proyecto deben realizar todas las acciones necesarias para que el titular de la institución apruebe y ordene publicar estos procedimientos en el menor tiempo posible.

Participantes: Analista de sistemas, Coordinador del Proyecto, Administrador del sistema.

Responsable: Analista de sistemas. 


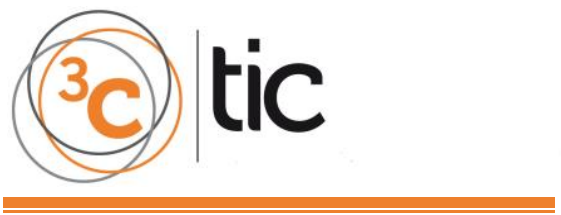

\section{Tarea ISI 5: Instalación del Sistema}

Esta tarea tiene como objetivo establecer el punto de inicio en que el sistema pasa a su instalación. Para ello es necesario que, se disponga del entorno del proceso perfectamente instalado en cuanto a hardware y software de base, componentes del nuevo sistema y procedimientos manuales y automáticos.

Participantes: Usuarios finales, Analista de Soporte Técnico, Analista de Sistemas, administrador del sistema.

Responsable: Administrador del Sistema.

\section{Tarea ISI 6: Puesta en marcha del sistema}

En esta actividad se pone en marcha el sistema y estará a cargo del equipo de Usuarios.

Participantes: Usuarios finales, Analista de Sistemas, Administrador del sistema.

Responsable: Usuarios finales.

\section{Tarea ISI 7: Reunión de trabajo para evaluación}

El objetivo de esta actividad es el evaluar el rendimiento del sistema, si cumple con las expectativas antes mencionadas en la formulación del Proyecto, de haber alguna modificación o ajuste a este proyecto, éste se realizará para mejora del sistema. En esta reunión se buscará la aprobación formal de la implantación del sistema por parte del Magistrado y el equipo de trabajo.

\section{PRUEBAS}

Las pruebas realizadas a la aplicación, previa a la implementación se derivaron en dos categorías: pruebas de validación y funcionales.

\section{Pruebas de validación}

Estas pruebas tienen como objetivo evaluar el cumplimiento de los requisitos de sistema, principalmente en los valores o datos que van a ser almacenados dentro de la base de datos, que cada uno de los campos tomen los valores correctos para su uso y manipulación.

\section{Pruebas funcionales}

Estas pruebas tienen como objetivo determinar el buen funcionamiento del sistema, comprobando la compatibilidad y funcionabilidad de las interfaces entre las distintas partes que conforman el sistema, cada una de ellas se evaluará de la siguiente forma: 


\section{Pruebas de usabilidad}

Estas pruebas determinan la facilidad del usuario para poder operar la aplicación. Como objetivos particulares, estas pruebas determinan si el usuario puede utilizar el sistema completando satisfactoriamente el proceso de búsqueda, otro de los puntos es comprobar si la interfaz es lo suficiente intuitiva para los usuarios que tengan experiencia en el manejo de este tipo de aplicaciones como para los que no la tienen y concluir si el sistema requiere de modificaciones para que se cumplan los objetivos anteriores.

\section{Pruebas de carga de datos}

Estas pruebas se le realizaron a la aplicación para comprobar los límites de carga de datos máxima que el sistema soporta en un momento determinado y así detectar a tiempo si hay algún problema por sobrecarga de información.

\section{Pruebas de desempeño}

El objetivo de esta prueba evalúa el desempeño del sistema en base a un tiempo de respuesta en las consultas para determinar si la aplicación es competente para operar dentro de un rango de tiempo aceptable y satisfacer las expectativas de los usuarios.

\section{Formato de pruebas}

En la tabla 1 se muestra el formato de pruebas que se utilizó para hacer una de las pruebas de funcionamiento en la vista del Proyectista, cada ITEM refiere a la prueba a ejecutar:

Tabla 1. Formato de pruebas, prueba funcional para vista de Proyectista.

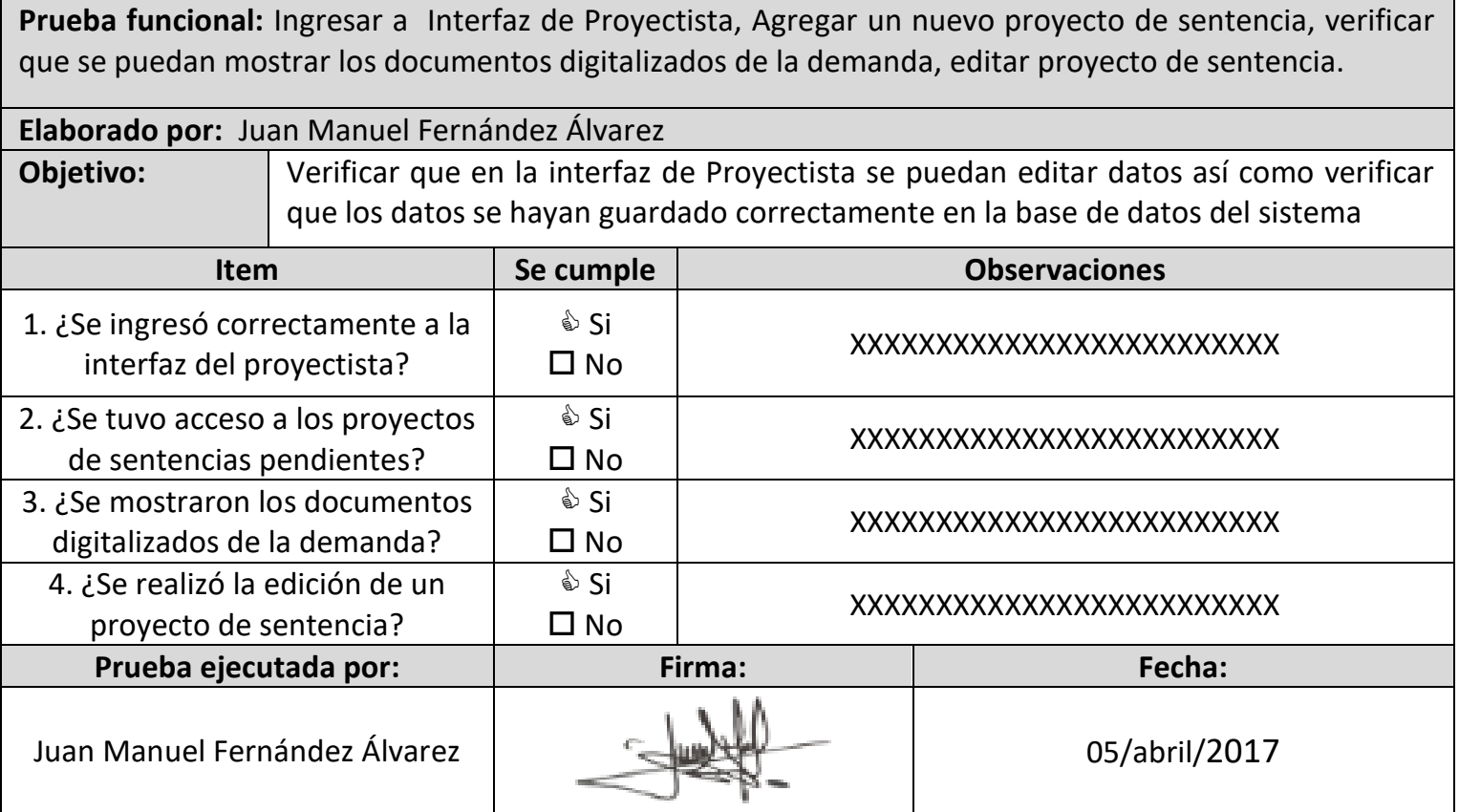

Fuente: elaboración propia. 


\section{RESULTADOS}

Después de haber realizado una codificación de cada una de las vistas se realizaron pruebas con el sistema de validación, verificación e instalación y se pudo observar que se cumple con los requerimientos anteriormente mencionados.

En la figura 9 se ilustra la vista lista de proyectos pendientes a realizar sentencia que llegan a cada uno de los proyectistas. Para poder comenzar la redacción, el proyectista realiza una lectura de los documentos que tienen relación con la demanda para así poder elaborar el proyecto de sentencia. En la figura 10 se ilustra la vista donde, tanto el proyectista como el magistrado pueden redactar, leer y/o editar las propuestas de sentencias que se han elaborado.

$\underline{T A}$ Tribunal de lo Contencioso Administrativo del Estado de
Colima

\begin{tabular}{|c|c|c|c|c|c|}
\hline \multicolumn{3}{|l|}{ SISeDe v1.0 } & Expedientes Pendientes 42 & Mi cuenta( Usuario ) . & Cerrar sesión \\
\hline & Expediente & Fecha & Acción a realizar & & \\
\hline & 0001 & $2016-11-21$ 02:02:00 & Ca & & \\
\hline & 0002 & $2016-11-2102: 03: 00$ & Cà & & \\
\hline & 0003 & $0000-00-0000-00: 00$ & & & \\
\hline
\end{tabular}

Figura 10. Lista de Proyectos Pendientes a elaborar. 


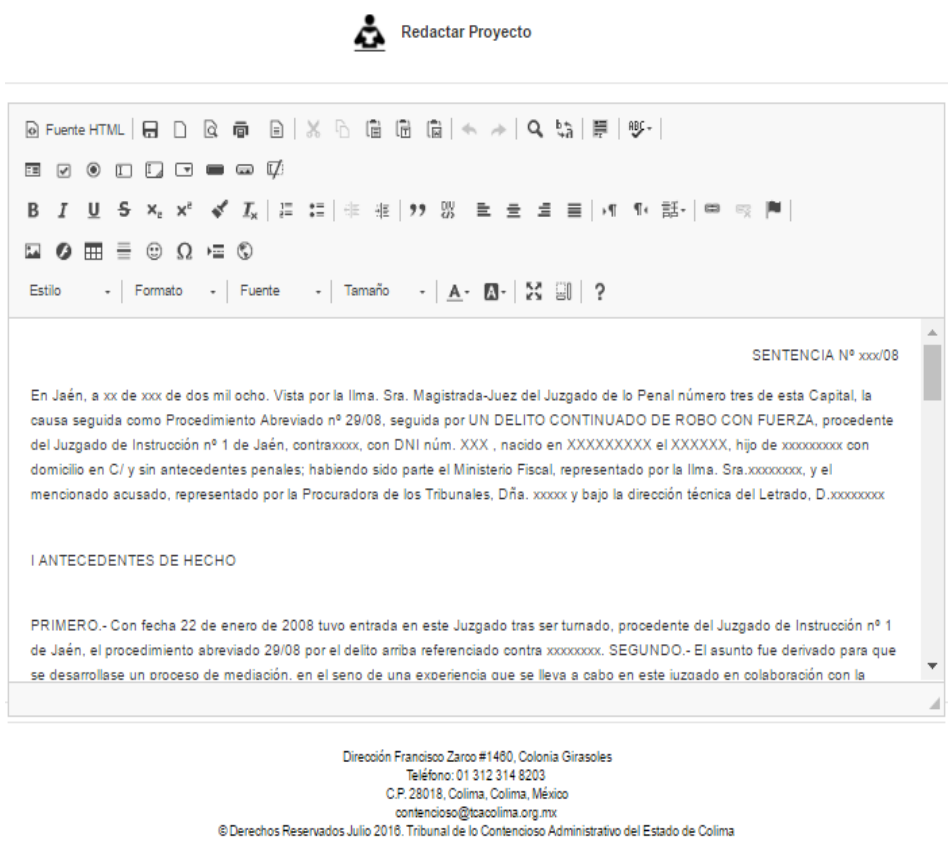

Figura 11. Vista redactar/editar proyecto de sentencia.

En la figura 11 se ilustran bitácoras para el registro histórico de las actividades utilizadas en el proceso de sentencias, ya que cada actividad tiene puntos de verificación para conocer el avance del litigio.

TA

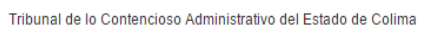

SISeDe v1.0

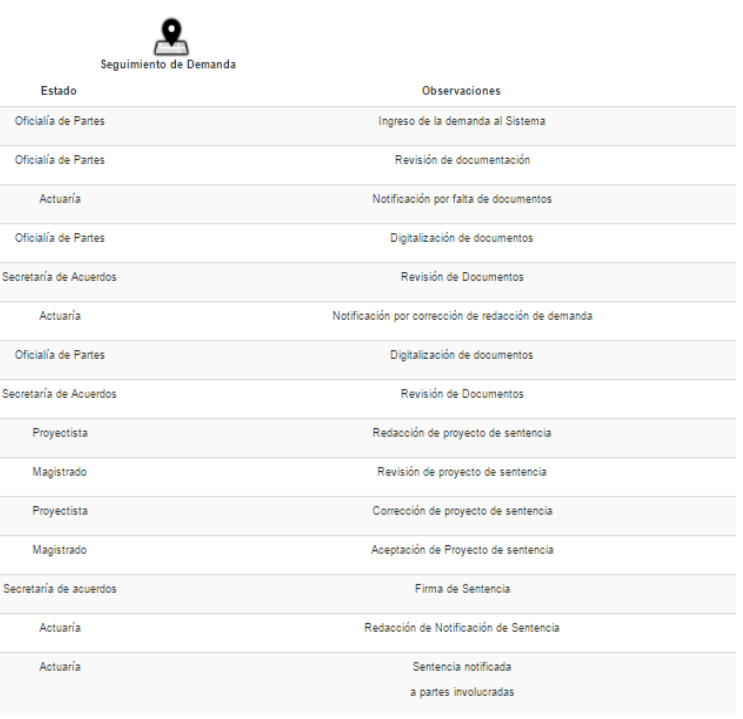

Figura 12. Seguimiento de demanda desde su ingreso hasta su notificación a partes involucradas. 


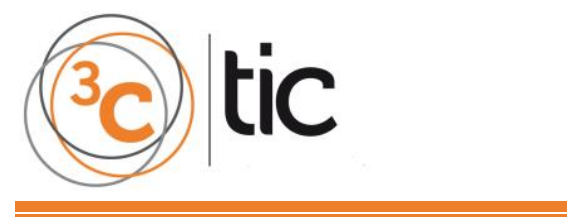

El uso de las TIC's en los procesos jurídicos administrativos contribuyen con una mejora en el proceso del litigio automatizando los procesos de seguimiento de proyectos de sentencias. Habitualmente, cada uno de los procesos que involucran a un proyecto de sentencia correspondiente a una demanda causaba un retraso en los tiempos de respuesta para poder dictar una sentencia definitiva para cada una de ella y espacio físico de almacenamiento.

\section{DISCUSIÓN Y CONCLUSIONES}

Este proyecto prevé un cambio total en el software, a través del cual el TCAEC realiza un seguimiento de los distintos trámites por los que va transitando un expediente judicial. Habilitada la gestión de sentencias con TIC's de vanguardia que favorecen la comunicación entre los actores que intervienen, se determinó que el desarrollo de un sistema web de seguimiento de sentencias mejora la administración de los procesos judiciales en el Tribunal de lo Contencioso Administrativo del Estado de Colima, ya que se desarrolló bajo un enfoque innovador utilizando bitácoras para el registro histórico de las actividades utilizadas en el proceso de sentencias. Se establecieron puntos de verificación para conocer el avance del litigio llevando el control del proceso en horas hombre, característica que los sistemas similares no tienen considerado.

Para continuar con este trabajo, se recomienda que cada uno de los documentos que se van a manejar en cada una de las partes involucradas en el proceso sean codificados para evitar el robo o alteración de cada uno de ellos ya que dentro de la seguridad informática ha habido muchos ataques cibernéticos para robo de información de empresas y personas físicas. 


\section{DISCUSIÓN Y CONCLUSIONES}

Gámez, A. M. (2015). Manual de Codeigniter.

Métodos de desarrollo Ágil. (2016). Obtenido de Proceso Unificado Agil (PUA): http://metodosdesarrolloagil.wikispaces.com/-+Proceso+Unificado+Agil+(PUA)

Mundo, R. d. (2005). Cumbre mundial sobre la Sociedad de la Información. Compromiso de Túnez. Ginebra.

Navas, G. Q. (2011). Contencioso Administrativo y medios electrónicos: un gran paso hacia la modernización del ejercicio de la justicia administrativa. Revista de Derecho, comunicaciones y Nuevas Tecnologías, 1-27.

TCAEC. (1996). Tribunal de lo Contencioso Administrativo del Estado de Colima. Obtenido de Presentación: http://tcacolima.org.mx/presentacion.php

Urdapilleta, M. C. (2015). Notas Generales del juicio en línea. PRAXIS de la Justicia Federal, 120. 